\title{
Mechanical Analysis and Strength Checking of Current Collector Failure in the Winding Process of Lithium-Ion Batteries
}

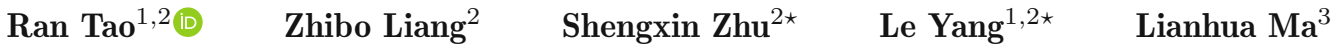 \\ Wei-li Song $^{2} \quad$ Haosen Chen ${ }^{1,2}$ \\ $\left({ }^{1}\right.$ AML, CNMM, Department of Engineering Mechanics, Tsinghua University, Beijing 100084, China) \\ ( ${ }^{2}$ Institute of Advanced Structure Technology, Beijing Institute of Technology, Beijing 100081, China) \\ $\left({ }^{3}\right.$ College of Civil Engineering and Architecture, Hebei University, Baoding 071002, China)
}

Received 21 September 2020; revision received 29 December 2020; Accepted 31 December 2020; published online 10 March 2021

(C) The Author(s) 2021

\begin{abstract}
The current collector fracture failure of lithium-ion batteries (LIBs) occurs during its winding production process frequently, and the consequent damages are usually large, but little research has been conducted on this phenomenon. This work stems from the difficulty and obstacles in the winding process of actual production of LIBs. The fracture failure of the current collectors is easily caused by the evolution and mutation of the mechanical behavior during the winding process, resulting in safety hazards and poor efficiency. The purpose of this work is to reveal the evolution and distribution mechanism of circumferential strain of the current collectors on the fracture failure under the constraint of winding process. Experimental tests, finite element calculations and theoretical model are used to study the evolution and distribution of circumferential strain. The dynamic evolution process of circumferential strain is tested accurately, and the mechanism of fracture failure of current collectors is revealed. The criterion for current collector strength is proposed based on the results of strain analysis and SEM observation.
\end{abstract}

KEY WORDS Lithium-ion batteries, Circumferential strain, Mechanical properties, Surface damage, Strength checking

\section{Introduction}

Lithium-ion batteries (LIBs) are a new type of rechargeable secondary batteries with many advantages such as high working voltage, high specific energy, long cycle life, no memory effect, no heavy metal and environmental friendliness [1-4]. As an energy storage power system, LIBs are widely used in hydraulic, thermal, wind and solar power stations, power tools, military equipment, aerospace and other fields [5-8]. At present, LIBs expand into emerging areas such as electric bicycles and electric vehicles gradually [9].

The rapid development of electric vehicles requires LIBs of higher productivity, higher energy density and lightweight [10-12]. Increasing the proportion of positive and negative active materials in the LIBs

\footnotetext{
* Corresponding authors. E-mails: zhusx19@163.com; leyang@pku.edu.cn
} 
is the most effective way to increase the specific energy density and reduce weight $[13,14]$. The high energy density and lightweight LIBs require electrodes that are thinner, longer, and have more turns $[15,16]$. Because the current collector of the LIBs is thin and long, the phenomenon of electrode breakage easily occurs during the winding process of the production $[17,18]$. This will increase the production cost of the LIBs, reduce production efficiency and pose a safety hazard $[19,20]$. Once the damaged electrode is wound into the LIBs, an increase in internal resistance causes the batteries to heat up, resulting in safety risk such as thermal runaway [21]. Research on mechanical strength of the current collectors winding is very urgent for high-performance and lightweight LIBs.

The fracture and failure of the current collectors are closely related to the mechanical properties of electrode materials and the evolution of the circumferential strain during winding [22]. Methods for accurately predicting circumferential strain are urgently needed. It is important to reveal the influence of the evolution behavior of circumferential strain on the fracture of current collectors, which is beneficial to improving and optimizing the production technology of winding process and enhancing production efficiency. However, there are currently very few studies on the effect of mechanical strain on fracture failure behavior in the winding of current collectors.

The copper and aluminum foils in the electrode are subjected to a rolling process [18]. A layer of electrode material is applied and rolled on the surface of copper and aluminum foils [23]. This process has an effect on the integrity and smoothness of the surface of copper and aluminum foils, but the effect on its mechanical properties is still unclear [19]. Bommel et al. [24] observed the morphology of the surface of the aluminum foil after wiping off the electrode material on the surface of the positive electrode by electron microscopy. The tensile mechanical properties of the surface-destroyed and rolled copper foil and aluminum foil are reduced. However, there is currently no research on the mechanism and regularity of the influence of winding process on the strength and damage of copper foil.

In this paper, the influence of the evolution of circumferential strain of current collectors during winding on the fracture failure is studied in the preparation process of cylindrical LIBs, and the design criteria for current collectors are proposed. The specific research is as follows. (1) The experimental platform characteristics of the winding process mechanics and the finite element model of the whole process of simulated winding are established. The stress values of the circumferential strain evolution process are measured in situ. (2) Based on the comparison between the results of experiments, numerical calculation and theoretical model, the mechanism of the circumferential strain's sudden increase when the current collector is wound onto the reel is explained. (3) The calibration formula of the current collector strength is established by the analytical results of circumferential strain during winding.

\section{Experimental Test Method}

Based on a lot of engineering technology research, we have found that the current collector structure has circumferential strain under the effect of tension in the actual winding production process of the high energy density and lightweight LIBs. The current collector is subjected to winding stress during the production, wherein the winding process is dynamically changing. The phenomenon of fracture failure easily occurs on the current collector of the LIBs during the dynamic winding process, which affects the production efficiency and brings safety hazards. In this work, a measuring apparatus of current collector winding is designed and manufactured to obtain the in situ circumferential strain during current collector winding. A schematic diagram of the winding apparatus for in situ measurement of circumferential strain is shown in Fig. 1. This work mainly conducts a winding test on the copper foil current collector. The tension at the time of winding the copper foil is measured by mounting a tension sensor on the device. The diameters of large and small reels are $40 \mathrm{~mm}$ and $20 \mathrm{~mm}$, respectively. The physical equipment of the winding device and the tension sensor can be found in Fig. 6 in "Appendix". The tension is measured by a tension sensor during the winding process. In Fig. 1, three strain gauges whose longitudinal directions are parallel to the direction in which the copper foil is stretched are attached to the surface of the copper foil, and the tensile strain of the outer surface of the copper foil is measured. The evolution of circumferential strain on the current collector surface during the winding process is obtained by measuring the surface strain of copper foil through strain gauges 1-3. During the winding process of copper foil, the circumferential stress in the elastic phase can be verified by the circumferential strain of the copper foil. One end of the copper foil is fixed to the large reel and a constant tension is applied to the other end. The winding machine rotates at a constant speed and 


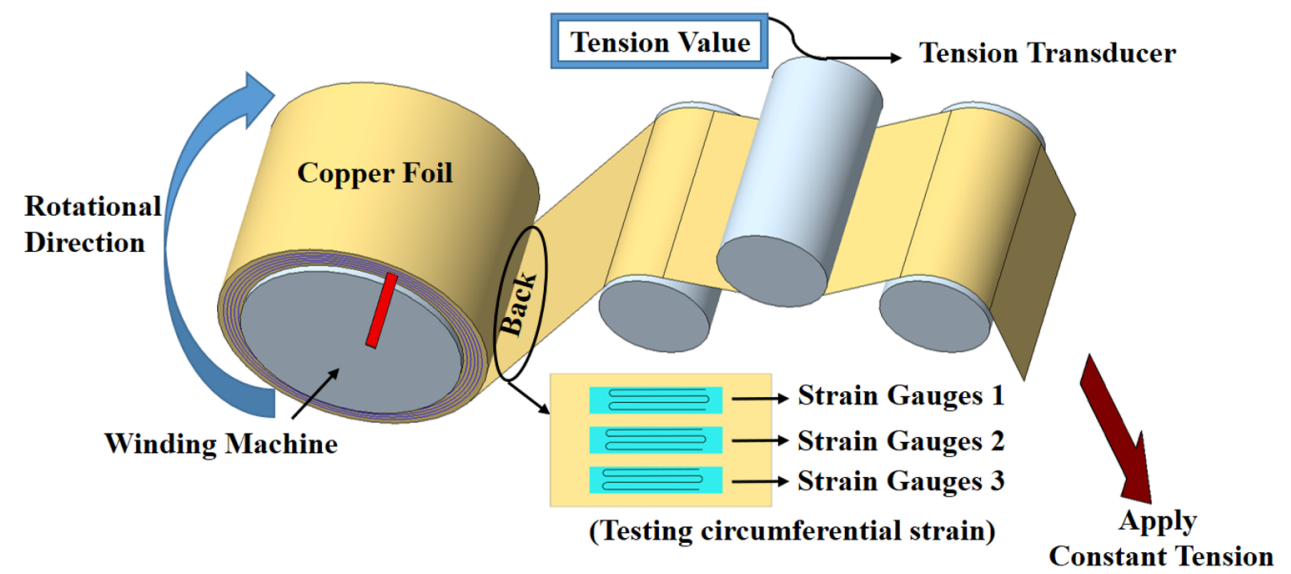

Fig. 1. A schematic diagram of the winding apparatus for in situ measurement of circumferential strain

a

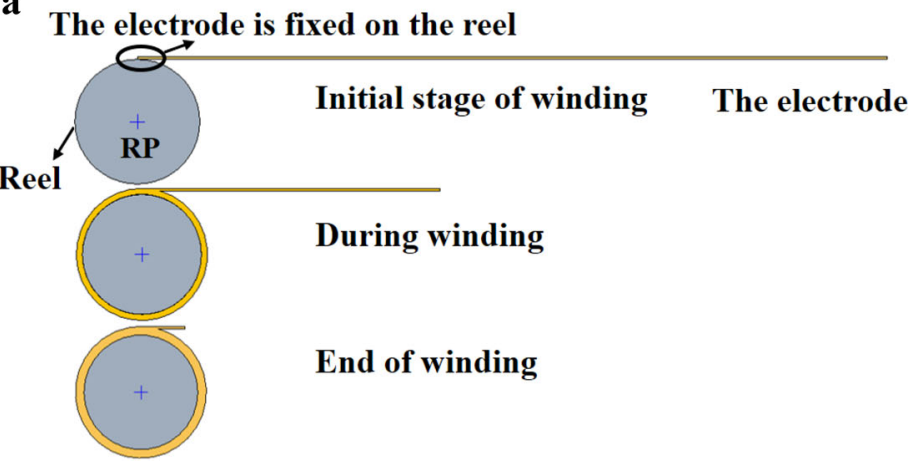

b

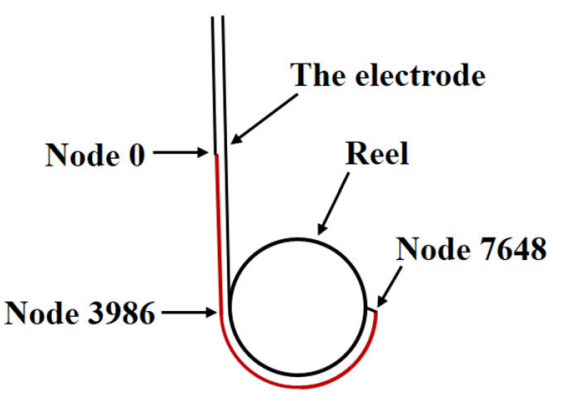

Fig. 2. a Schematic diagram of the current collector winding process. b Schematic diagram of the node position on the current collector surface

the pulling force is constant. Based on the DH3816N static strain test system and DHDAS software, the strain evolution of the copper foil during the winding process can be obtained.

\section{Finite Element Analysis}

Based on the nonlinear finite element software ABAQUS, the finite element calculation and analysis of the dynamic evolution of the circumferential strain during the winding process is implemented. The finite element model is divided into two parts: the reel part and the current collector part. The current collectors are two-dimensional solid elements, and the reel is a rigid body. To compare with the experimental results, the component size in simulation is consistent with the dimensions of the actual experimental components. The diameter of the reel is $40 \mathrm{~mm}$, and the thickness of the current collector can be set based on the actual product. Figure 2a is the simulation process diagram of the model. Because the coated electrode material has little effect on the elastic modulus of the current collector, the electrode material on the surface of the current collector is omitted in this simulation, and the mechanical properties of the current collector are determined by the elastic modulus and Poisson's ratio of the copper foil. Based on the tensile test of the copper foil, the elastic modulus and Poisson's ratio of current collector are $110 \mathrm{GPa}$ and 0.33 , respectively. This work divides the winding process into the following three steps. (1) One end of the collector is subjected to a constant pulling force, and the other end is fixed to the reel. (2) The reel sets the rotation displacement at a constant speed. (3) The rotation of the reel is canceled, and both ends of the current collector are fixed after winding. Using the static general algorithm, the large deformation is turned on to calculate the entire winding process. Subsequently, finite element calculations of the current collector winding processes of different thicknesses and tensions are carried out, and the circumferential strain evolution process is obtained. 

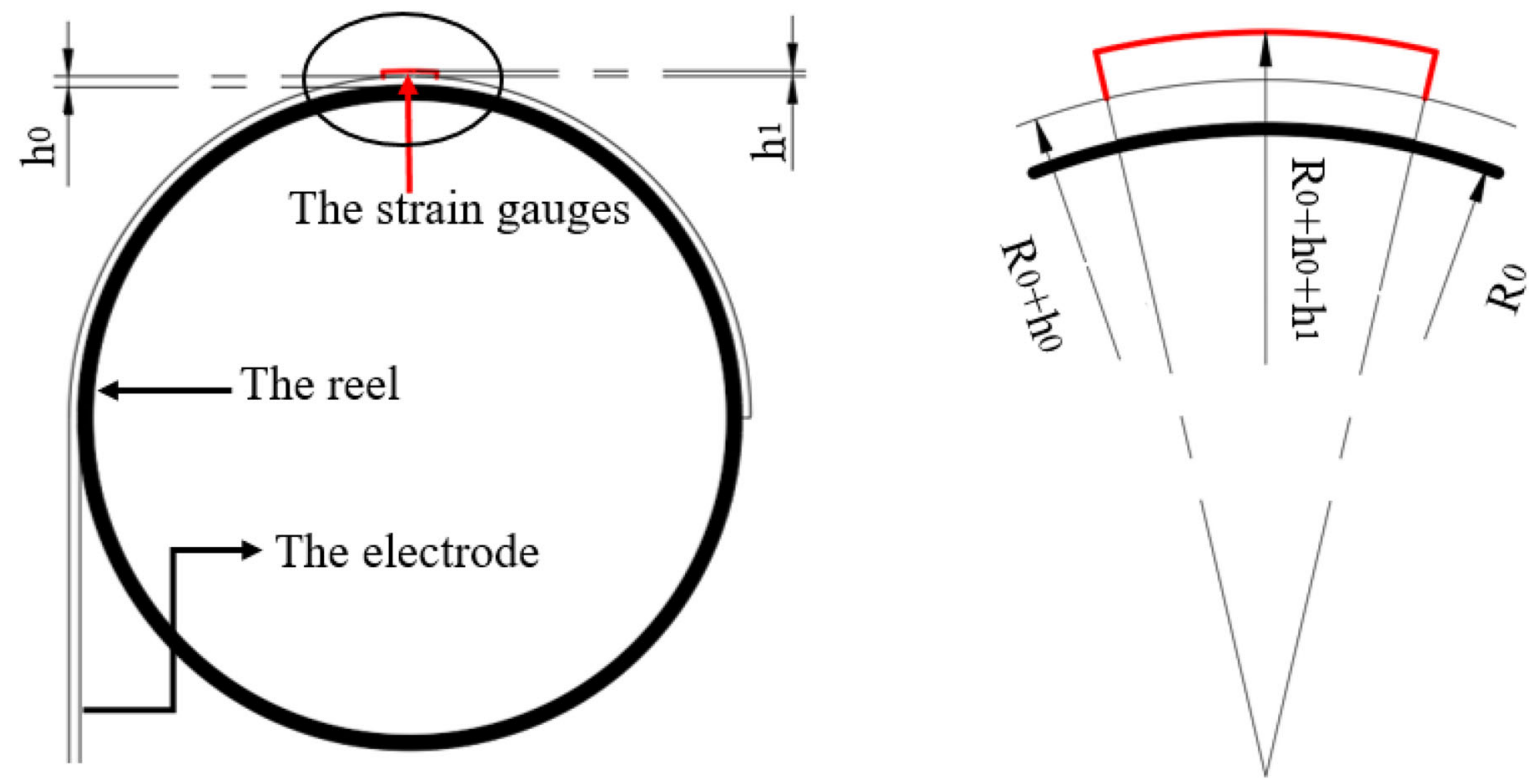

Fig. 3. Schematic diagram of correction principle of circumferential strain measured by strain gauges

At each time step, the stress at a fixed point on the current collector is extracted. The entire extracted circumferential strain region is the red node region in Fig. $2 \mathrm{~b}$. Thereby, the evolution process of the circumferential strain during winding is obtained by the simulation.

\section{Analysis and Discussion of Winding Strain}

Based on the analysis of the test results, we found that the strain measured by the strain gauges has a large error when the current collector is wound onto the reel (see Fig. 7 in "Appendix"). The strains measured by the strain gauges are those generated by the outer surface of the actual substrate bonded by the glue on the copper foil. When the copper foil is wound on the reel, tensile strain and bending strain exist on the outer surface of the copper foil, and the outer strain of the actual substrate produces a bending strain greater than that of the copper foil surface. Therefore, the strain gauges measured at the time of winding are larger than the strain generated by the outer surface of the copper foil. The circumferential strain measured by the strain gauges at the time of winding needs to be corrected. In Fig. 3, the radius of the reel is $R_{0}$, the thickness of the copper foil is $h_{0}$, and the thickness of the current collector sheet is $h_{1}$. Based on the above analysis and the strain correction schematic diagram, the relationship between the strain measured in the winding section strain gauge and the actual strain generated on the copper foil surface by the geometric principle is as follows

$$
\frac{R_{0}+h_{0}+h_{1}}{R_{0}+h_{0}}=\frac{l\left(1+\varepsilon_{1}\right)}{l\left(1+\varepsilon_{0}\right)}
$$

where $l, \varepsilon_{0}$ and $\varepsilon_{1}$ are the length of the strain gauge, the strain measured by the strain gauge and the real strain generated on the surface of the copper foil, respectively. The true strain generated on the surface of the current collector can be obtained by correcting the strain measured by the corresponding variation of Eq. (1). By Eq. (1), the true strain of the surface of the copper foil measured by the strain gauges $1-3$ in the winding section is as follows

$$
\varepsilon_{0}^{1}=\frac{\left(1+\varepsilon_{1}\right)\left(R_{0}+h_{0}+h_{1}\right)}{R_{0}+h_{0}}-1
$$

The thicknesses of the copper foil and the three strain gauges are measured by a digital dial gauge. Based on Eq. (2) and strains of the strain gauges 1-3 measured in the winding section, the real strain generated on the surface of the copper foil is obtained. 

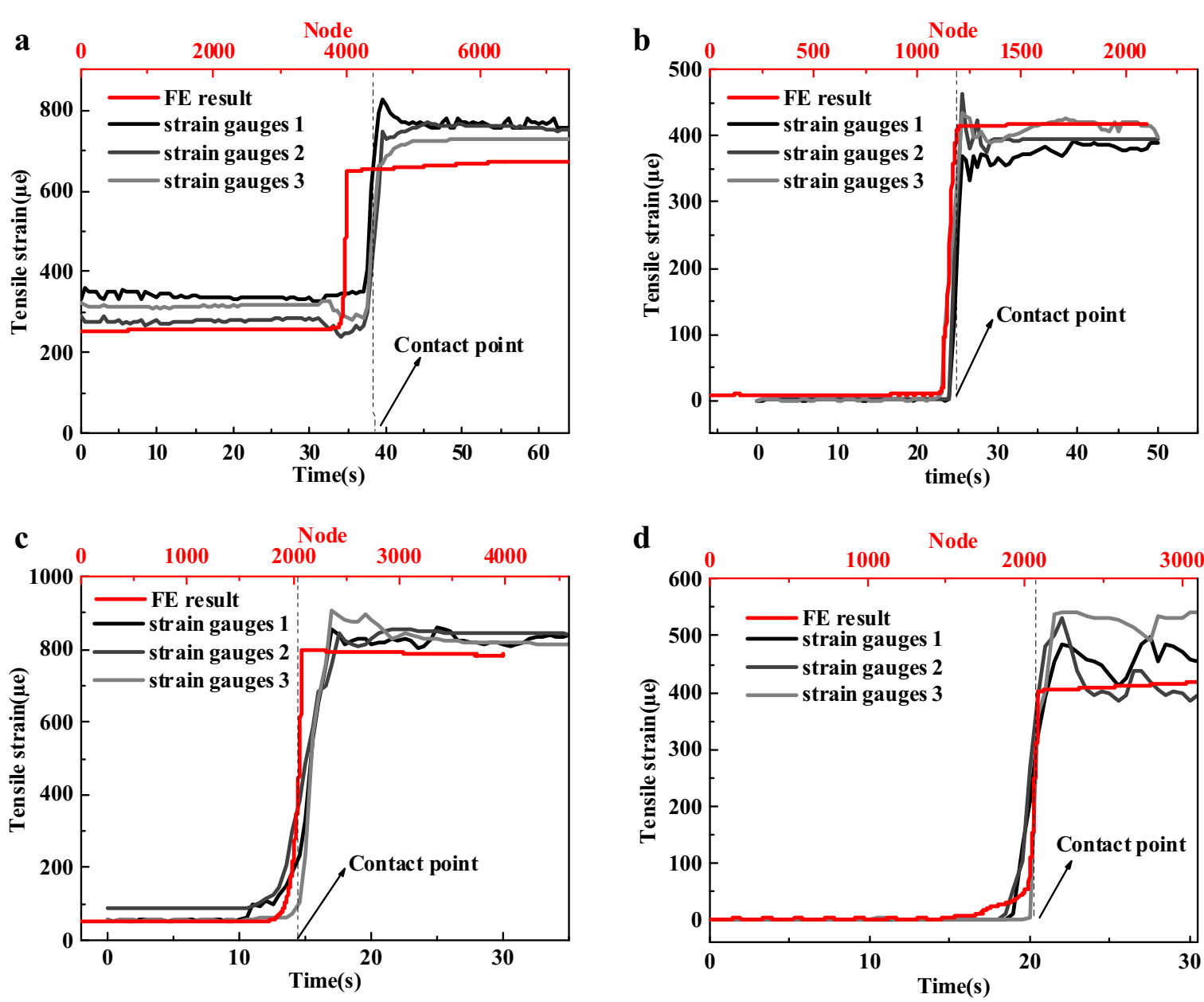

Fig. 4. The curves of circumferential strain evolution of different thicknesses and tensions based on finite element calculations and experimental tests: a the thickness of the copper foil is $60 \mu \mathrm{m}$, the tensile force is $20 \mathrm{MPa}$; $\mathbf{b}$ the thickness of the copper foil is $50 \mu \mathrm{m}$, the tensile force is $0.2 \mathrm{MPa}$; $\mathbf{c}$ the thickness of the copper foil is $100 \mu \mathrm{m}$, the tensile force is 5 $\mathrm{MPa}$; $\mathbf{d}$ the thickness of the copper foil is $100 \mu \mathrm{m}$, the tensile force is $0.2 \mathrm{MPa}$

From the results of actual circumferential strain in Fig. 4a, it is understood that the copper foil is subjected to a tensile stress of $20 \mathrm{MPa}$ and the circumferential strain is about $300 \mu \varepsilon$ when the copper foil with a thickness of $60 \mu \mathrm{m}$ is a straight line segment. In the three sets of comparisons, the experimental results in the straight line of the unwound copper foil strain are consistent with the simulation results. When the copper foil is wound from the straight section to the reel, the strain on the outer surface of the copper foil increases to about $700 \mu \varepsilon$ suddenly. In the simulation results, the circumferential tensile strain on the surface of the copper foil is about $260 \mu \varepsilon$ in the straight section before the copper foil is wound, and the circumferential tensile strain on the outer surface of the copper foil suddenly increases to $670 \mu \varepsilon$ when the copper foil is wound onto the reel. The experimental circumferential strain is slightly larger than the simulation one because the tensile force exerted on the copper foil is not sufficiently uniform in the experiment. In the LIBs' winding experiments, the trend of the experimental results of the evolution of circumferential strain is basically consistent with that of the simulation results.

To further verify the correctness of this correction method, we implement another three sets of experiments and simulations. When the thickness of the copper foil is $50 \mu \mathrm{m}$ and the tensile force is 0.2 $\mathrm{MPa}$, the experimental and simulation results of the tensile strain on the surface of the copper foil are shown in Fig. 4b. The modified experimental results of the circumferential strain evolution are basically consistent with the simulation results. When the thickness of the copper foil is $100 \mu \mathrm{m}$ and the tensile 
force is $5 \mathrm{MPa}$, the copper foil is in a straight line before winding and the tensile strain on the surface is $50 \mu \varepsilon$. When wound onto a reel, the tensile strain on the outer surface increases to about $800 \mu \varepsilon$ suddenly. The corrected experimental results are basically consistent with the simulation results. When the thickness of the copper foil is $100 \mu \mathrm{m}$ and the tensile force is $0.2 \mathrm{MPa}$, the experimental results are basically consistent with the simulation results.

In Fig. 4a-d, the comparison between experimental and simulation results verifies the accuracy of this correction method. When the current collector is wound onto the reel, the circumferential strain and tensile strain on the surface of the current collector suddenly increase. Therefore, the current collector surface will become a dangerous point for electrode failure when the electrode is just wound onto the reel.

Experimental, numerical and theoretical studies and analyses are performed on the evolution behavior of circumferential strain of the wound current collector. In Fig. 4, the experimental, numerical and theoretical results are consistent. In this work, the experimental methods, numerical simulation methods and theoretical models developed can effectively predict the circumferential strain at different thicknesses and tensions. These efforts can provide reference and support for further optimization of LIBs' winding production. In addition, the design criteria for the damage of the current collector of the winding process should be proposed based on the results of the circumferential strain study.

\section{Criterion for Electrode Strength}

To determine whether the strength of the current collectors is sufficient, we need to establish a strength criterion during the winding process of current collectors. According to the strength criterion, the thickness of the current collectors, tension and reel shape can be designed and screened to ensure that the current collectors can be wound safely and normally. The strength criterion formula of copper foil is as

$$
\sigma_{\max } \leq[\sigma]
$$

where $\sigma_{\max }$ is the maximum tensile stress generated by the copper foil in a straight line before winding, and $[\sigma]$ is the safe allowable stress of the copper foil. When the copper foil is wound onto the reel, the circumferential strain on the outer surface of the copper foil increases suddenly. At this time, the maximum stress of the copper foil is no longer the uniform tensile stress generated when the straight section is stretched, but the tensile stress generated on the outer surface of the winding section. The outer surface of the copper foil becomes a dangerous point for strength failure. Thus, the strength criterion of the copper foil is corrected to

$$
k_{1} \sigma_{\max } \leq[\sigma]
$$

where $k_{1}$ is the weighting coefficient. The copper foil is subjected to both tensile stress and bending stress in the winding part, so the tensile stress of the winding part is much larger than the tensile stress generated in the straight section.

The copper foil is subjected to a rolling process, and its surface is rolled by a layer of the electrode material, which may cause damage to the surface of the copper foil. The microscopic topography of the copper foil surface before and after rolling is obtained by the scanning electron microscopy (SEM). In Fig. 5a, the surface of the copper foil which is not pressed by the active material is mostly smooth, with only small pits. Figure 5b shows an SEM image of the surface morphology of a copper foil after rolling a graphite-based active material. Figure $5 \mathrm{~b}$ shows the microstructure of a graphite-based active material rolled on the surface of the electrode, in which it can be seen that the material is very compact by rolling. Subsequently, the active material on the surface of the copper foil is removed by n-methylpyrrolidone (NMP) wiping and ultrasonic cleaning. Figure 5c shows the SEM image of the surface of cleaned current collector copper foil. It is found that the surface of the copper foil after the rolling process is obviously damaged, and most of the flat surface becomes pitted. Because the surface of the copper foil is damaged by the rolling process and the added active material has little effect on the tensile mechanical properties, the tensile strength of the current collectors is lower than that of the copper foil. This damage affects the mechanical properties of the current collector and reduces its allowable strength. 
$\mathbf{a}$
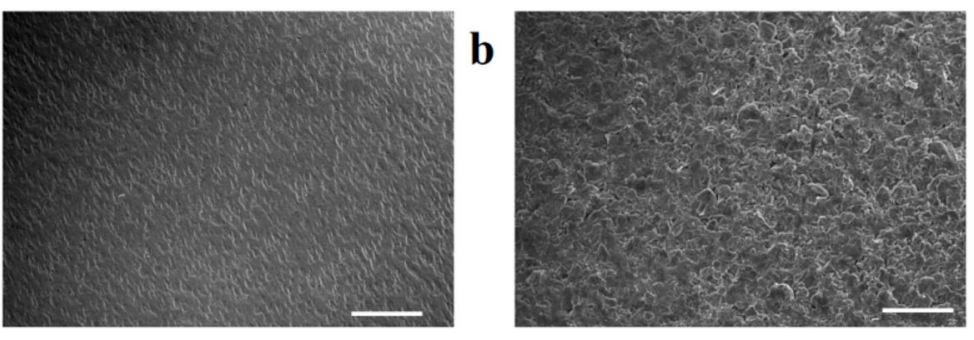

d
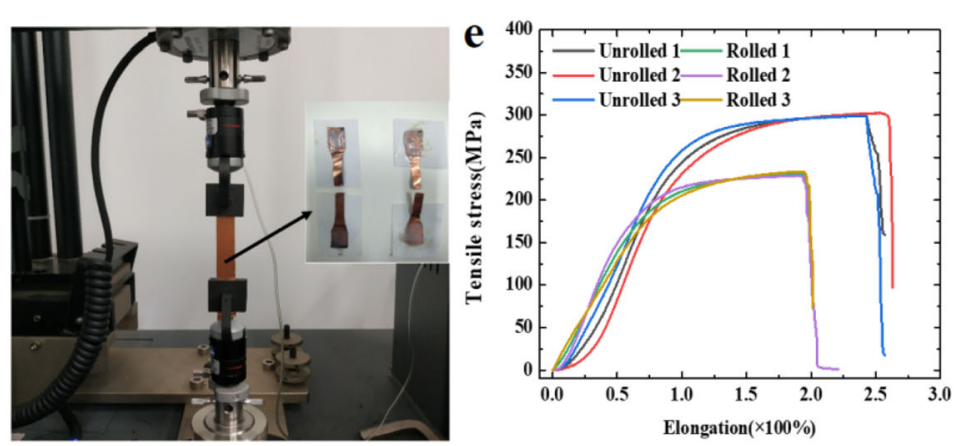
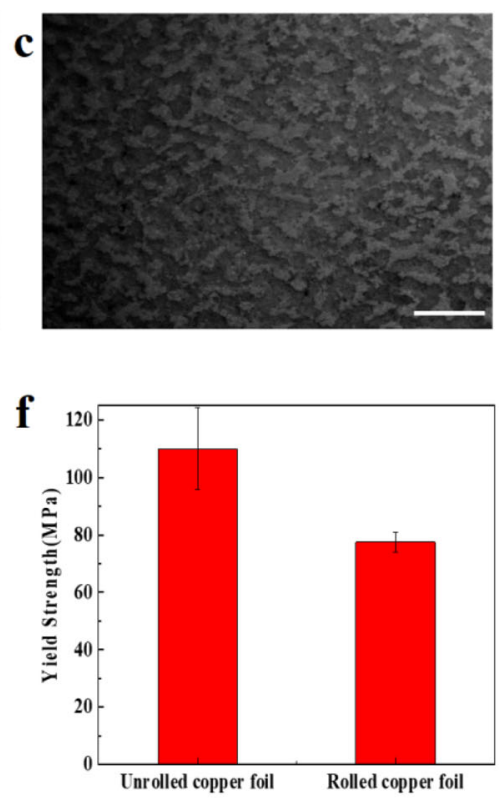

Fig. 5. SEM image of surface on the copper foil current collector and tensile strength test: a the surface of the current collector foil; $\mathbf{b}$ the surface of the current collector after rolling the active material; $\mathbf{c}$ the surface of the copper foil current collector after the active material is removed; $\mathbf{d}$ tensile strength test of copper foil current collector; e strength test curve; f comparison of yield strength between copper foil and unrolled copper foil after rolling (scale bar, $80 \mathrm{um}$ )

Two sets of specimens are prepared, each containing three samples. One set is a copper foil that is not rolled with active material. The other set is an electrode, in which the electrode material on the surface of the negative electrode is wiped clean by NMP. Based on the standard tensile test method for metal foil, two sets of copper foils are separately made into stretched standard parts. The schematic diagram of the dimensions of the stretched sample is shown in Fig. 8 of "Appendix". Figure 5d displays the tensile test of two sets of copper foils and the sample pieces after failure. Figure 5e shows the tensile stress-elongation curve and breaking strength of the two sets of specimens in the tensile test. The experimental results show that the mechanical properties of the rolled electrode are much worse than the unrolled copper foil. The yield strengths of the copper foil before and after rolling are compared, as shown in Fig. 5f. The allowable stress of the rolled copper foil is lower than the allowable stress of the unrolled copper foil. Therefore, the copper foil strength check formula of Eq. (4) can be corrected as

$$
k_{1} \sigma_{\max } \leq k_{2}[\sigma]
$$

where $k_{2}$ is a weighting coefficient of the allowable stress $[\sigma]$ of the copper foil. According to the data in Fig. 5f, the value of the weighting coefficient $k_{2}$ is about 0.7 . The design criteria for the failure of the current collectors during the winding process proposed in this work can provide guidance for the winding process and production of LIBs.

\section{Conclusions}

The paper reveals the influence of the evolution and distribution of circumferential strain on the fracture failure under the constraints of the winding process. Experimental tests, finite element calculation and theoretical analysis are used to test and analyze the evolution and distribution of circumferential strain. The criterion for current collector strength is formulated based on the mechanical properties of electrodes and the phenomenon of SEM observation. The conclusions from these studies can be summarized as follows. 
(1) Based on the current collector winding apparatus and the analysis formula, the experimental test method for the circumferential strain of the electrode is obtained, and the correctness of the experimental method is verified by comparison with the finite element method.

(2) The dynamic evolution and the mutation behavior of circumferential strain of the current collector are quantitatively characterized, and its influence on the fracture failure of the current collector is revealed.

(3) The microscopic appearance of the damaged surface of the copper foil by the smeared and rolled electrode material is demonstrated based on SEM.

(4) The criterion for the current collector strength is established as a reference for the winding process of LIBs.

Acknowledgements. National Natural Science Foundation of China (12002183), Postdoctoral Science Foundation (2019TQ0171, 2020M670304) and Beijing Natural Science Foundation (Grant Nos. 16L00001 and 2182065) are gratefully acknowledged.

Open Access This article is licensed under a Creative Commons Attribution 4.0 International License, which permits use, sharing, adaptation, distribution and reproduction in any medium or format, as long as you give appropriate credit to the original author(s) and the source, provide a link to the Creative Commons licence, and indicate if changes were made. The images or other third party material in this article are included in the article's Creative Commons licence, unless indicated otherwise in a credit line to the material. If material is not included in the article's Creative Commons licence and your intended use is not permitted by statutory regulation or exceeds the permitted use, you will need to obtain permission directly from the copyright holder. To view a copy of this licence, visit http://creativecommons.org/ licenses/by/4.0/.

\section{References}

[1] Jiao S, Ren X, Cao R, Engelhard MH, Liu Y, Hu D. Stable cycling of high-voltage lithium metal batteries in ether electrolytes. Nat Energy. 2018;3(9):739-46.

[2] Griffith KJ, Wiaderek KM, Cibin G, Marbella LE, Grey CP. Niobium tungsten oxides for high-rate lithium-ion energy storage. Nature. 2018;559(7715):556.

[3] Rustomji CS, Yang Y, Kim TK, Mac J, Kim YJ, Caldwell E. Liquefied gas electrolytes for electrochemical energy storage devices. Science. 2017;356(4263):6345.

[4] Manikkavel A, Kumar V, Lee DJ. Simple fracture model for an electrode and interfacial crack in a dielectric elastomer under tensile loading. Theor Appl Fract Mech. 2020;108:102626.

[5] Schiffer ZJ, Arnold CB. Characterization and model of piezoelectrochemical energy harvesting using lithium ion batteries. Exp Mech. 2018;58:605-11.

[6] Jones EMC, Silberstein MN, White SR, et al. In Situ measurements of strains in composite battery electrodes during electrochemical cycling. Exp Mech. 2014;54(6):971-85.

[7] Chang C, Li X, Xu Z, Gao H. Lithiation-enhanced charge transfer and sliding strength at the silicongraphene interface: a first-principles study. Acta Mech Solida Sin. 2017;30:254-62.

[8] Kespe M, Nirschl H. Numerical simulation of lithium-ion battery performance considering electrode microstructure. Int J Energy Res. 2015;39(15):2062-74.

[9] Lyu Z, Gao R. A model-based and data-driven joint method for state-of-health estimation of lithium-ion battery in electric vehicles. Int J Energy Res. 2019;43:7956-69.

[10] Golozar M, Hovington P, Paolella A, Bessette S, Lagace M, Bouchard P. In situ scanning electron microscopy detection of carbide nature of dendrites in li-polymer batteries. Nano Lett. 2018;18(12):7583-9.

[11] Lu Y, Ni Y. Stress-mediated lithiation in nanoscale phase transformation electrodes. Acta Mech Solida Sin. 2017;30:248-53.

[12] Sieg J, Bandlow J, Mitsch T, Dragicevic D, Materna T, Spier B. Fast charging of an electric vehicle lithium-ion battery at the limit of the lithium deposition process. J Power Sources. 2019;427:260-70.

[13] Sun H, Mei L, Liang J, Zhao Z, Lee C, Fei H. Three-dimensional holey-graphene/niobia composite architectures for ultrahigh-rate energy storage. Science. 2017;356(6338):599-604.

[14] Liu Y, Xiong L, Li P, Fu H, Hou Z, Zhu L. Self-supported CuO nanoflake arrays on nanoporous $\mathrm{Cu}$ substrate as high-performance negative-electrodes for lithium-ion batteries. J Power Sources. 2019;428:206.

[15] Li M, Lu J, Chen Z, Amine K. 30 years of lithium-ion batteries. Adv Mater. 2018;30:180056133.

[16] Kang J, Rizzoni G. Study of relationship between temperature and thermal energy, operating conditions as well as environmental factors in large-scale lithium-ion batteries. Int J Energy Res. 2014;38(15):1994-2002.

[17] David L, Bhandavat R, Barrera U, Singh G. Silicon oxycarbide glass-graphene composite paper electrode for long-cycle lithium-ion batteries. Nat Commun. 2016;7(10998):1-10. 
[18] De Vasconcelos LS, Sharma N, Xu R, et al. In-situ nanoindentation measurement of local mechanical behavior of a Li-Ion battery cathode in liquid electrolyte. Exp Mech. 2018;59:337-47.

[19] Chen C, Zhang Y, Li Y, Kuang Y, Song J, Luo W. Highly conductive, lightweight, low-tortuosity carbon frameworks as ultrathick 3D current collectors. Adv Energy Mater. 2017;17(7):1700595.

[20] Chen M, Liu J, Ouyang D, Wang J. Experimental investigation on the effect of ambient pressure on thermal runaway and fire behaviors of lithium-ion batteries. Int J Energy Res. 2019;43(9):4898-911.

[21] Zhang S, Xu K, Read J. A non-aqueous electrolyte for the operation of Li/air battery in ambient environment. J Power Sources. 2011;196(8):3906-10.

[22] Wang M, Tang M, Chen S, Ci H, Wang K, Shi L. Graphene-armored aluminum foil with enhanced anticorrosion performance as current collectors for lithium-ion battery. Adv Mater. 2017;29(1703882):1-7.

[23] Li L, Bian Y, Zhang X, Xue Q, Fan E, Wu F. Economical recycling process for spent lithium-ion batteries and macro- and micro-scale mechanistic study. J Power Sources. 2018;377:70-9.

[24] Van Bommel A, Divigalpitiya R. Effect of calendaring $\mathrm{LiFePO}_{4}$ electrodes. J Electrochem Soc. 2012;159(11):A1791-5.

\section{Appendix}

The winding device is customized by CAD drawing, and the radius of the reel is $40 \mathrm{~mm}$. The tension at the winding of the copper foil is measured by mounting a tension sensor next to the reel. The winding process is performed by manually turning the handle, and a constant tension during winding is applied by gravity. The physical equipment of the winding device and the tension sensor is shown in Fig. 6 .

The tension sensor is manufactured by Sensor Systems Engineering Co., Ltd. and the model number is JZHL-3. The technical parameters of the tension sensor are listed in Table 1.

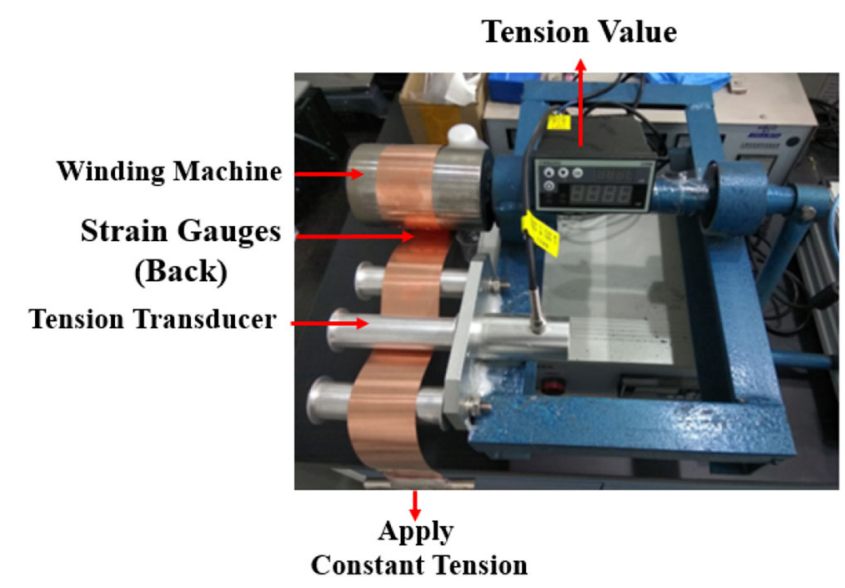

Fig. 6. The physical equipment of the winding device and the tension sensor

Table 1. Technical parameters of the tension sensor

\begin{tabular}{ll}
\hline Technical name & Parameter \\
\hline Sensor model & JZHL-3 \\
Range & $0-200 \mathrm{~N}$ \\
Overrange & 1.2 times \\
Precision & $\pm 0.05 \%$ \\
Material & Aluminum alloy \\
Configurable output device & Digital interface RS485/RS232 \\
\hline
\end{tabular}




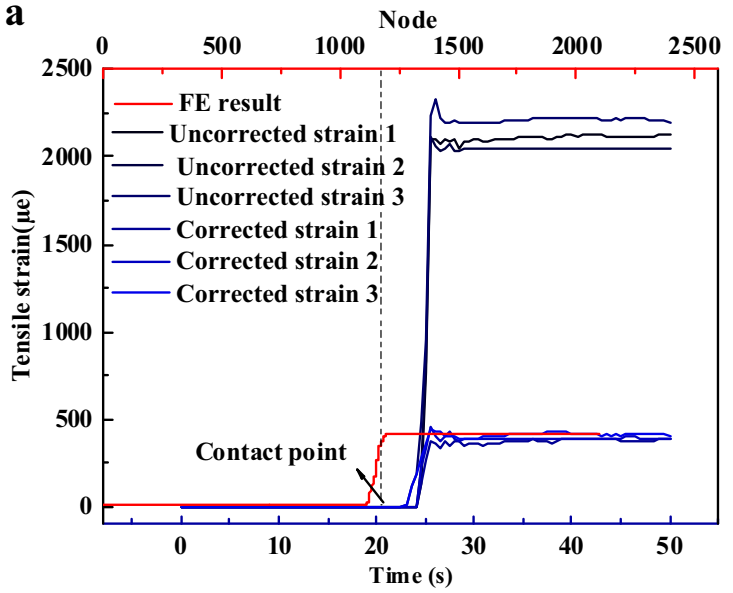

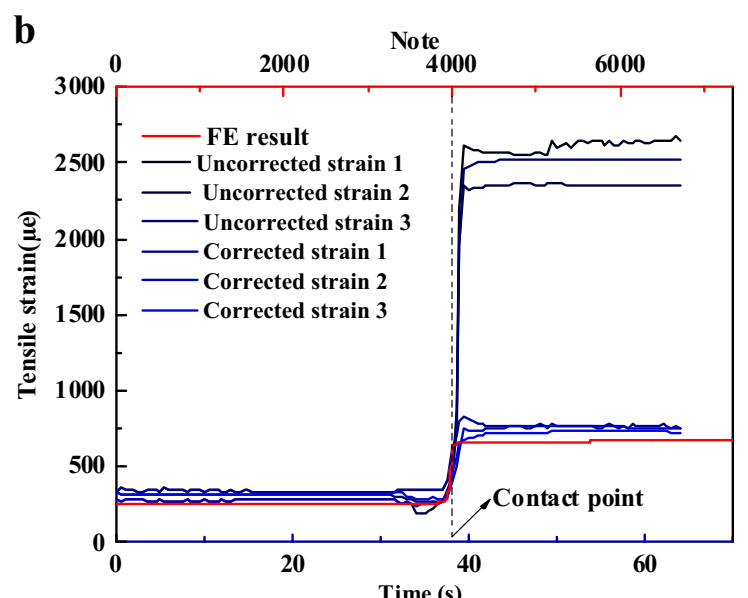

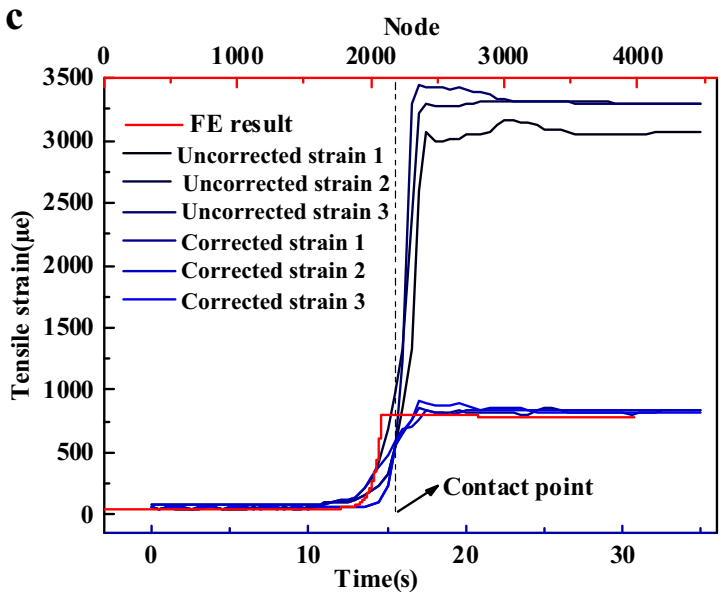

Fig. 7. The curves of circumferential strain evolution with time in different thicknesses and tensions based on finite element calculations, uncorrected and corrected experimental tests: a the thickness of the copper foil is $60 \mu \mathrm{m}$, the tensile force is $20 \mathrm{MPa}$; $\mathbf{b}$ the thickness of the copper foil is $50 \mu \mathrm{m}$, the tensile force is $0.2 \mathrm{MPa}$; c the thickness of the copper foil is $100 \mu \mathrm{m}$, the tensile force is $5 \mathrm{MPa}$

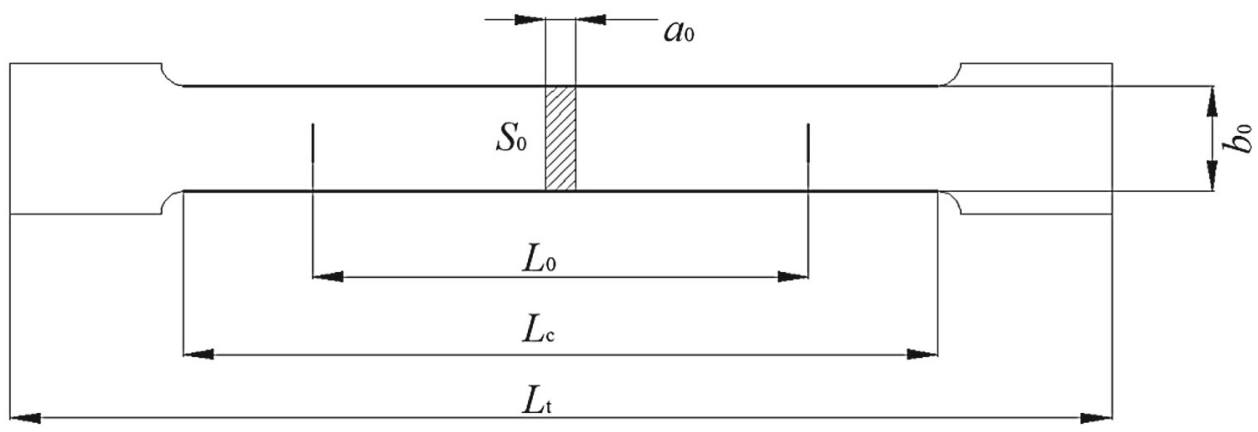

Fig. 8. Schematic diagram of the stretched sample size $a_{0}$ is the thickness of the sample; $b_{0}$ is the parallel part width of the sample, which is $12.5 \mathrm{~mm} ; S_{0}$ is the experimental original cross-sectional area; $L_{0}$ is the experimental original gauge length, which is $50 \mathrm{~mm} ; L_{\mathrm{c}}$ is the parallel part length of the sample, which is $60 \mathrm{~mm}$; and $L_{\mathrm{t}}$ is the total length of the sample, which is $120 \mathrm{~mm}$ 